\title{
La carne y lo irreversible del cuerpo pulsional
}

\author{
The Flesh and the irreversibility of \\ the body in the domain of the drive
}

\section{Bruno Carignano}

\section{RESUMEN:}

Con una metodología teórica examinamos a la visión comprendida en el espacio merleau-pontyano; el objetivo es el de interrogar al excedente del cuerpo respecto del imaginario especular. La reversibilidad malograda entre lo activo y lo pasivo que allí se vislumbra nos sirve de fundamento para circunscribir al cuerpo pulsional en su anclaje fantasmático. Nos apoyamos en la distinción establecida por Merleau-Ponty entre la carne del cuerpo y la carne del mundo para dilucidar la negatividad que trabaja al cuerpo pulsional. La interferencia de la alteridad permite contraponerlo al modo del cuerpo desprendido de su ontología de la carne, cuya réplica él pesquisa en teorizaciones de Melanie Klein. Tomando en cuenta estas consideraciones, nos dirigimos a algunos desarrollos de Freud y Lacan para despejar la disparidad de los cuerpos pulsional y narcisista: al eclipse, en el campo del amor narcisista, de las dimensiones del Otro y del objeto causa puede contraponerse el tratamiento del objeto característico del plano pulsional. El límite autoerótico de la pulsión, figurado en la reversibilidad consumada de la carne, ofi-

\section{SUMMARY:}

The methodology of this paper is theoretical. Our main objective is to examine the vision in Merleau-Ponty's space, in order to interrogate the body beyond the specular structure of the imaginary. The failure of identity between activity and passivity can be used as a ground to analyze the body on the axis drive-phantasy. We examine how the notion of a failed reversibility can be related to the different senses of the Flesh, and we interrogate the body that is determined by the negativity of the drive. That leads us to consider the difference with the ontology of the Flesh deduced in Melanie Klein's theory by Merleau-Ponty. Afterwards, we examine some crucial distinctions made by Freud and Lacan. They allow us to distinguish, from the point of view of the object, the body in the narcissistic field of love from the body in the domain of the drive: while in the former there is a dissolution of both the dimensions of the Other and the object as cause of desire, in the latter we face the transcendence of the object. Finally, we consider the theoretical importance of the autoerotic limit of the drive, which can be 
cia de modelo que tomamos finalmente en consideración para exponer, por contraste, el estatuto de la pulsión parcial.

\section{Palabras Clave:}

Cuerpo - carne - narcisismo - pulsión - imaginario illustrated with the accomplishment of the reversibility of the Flesh.

\section{WORD PAD:}

Body - Flesh - Narcissism - Drive Imaginary 
Reversibilidad siempre inminente $y$ jamás realizada de hecho M. Merleau-Ponty

\section{Objeto intragable}

J. LACAN

\section{INTRODUCCIÓN \\ EL ESPACIO TOPOLÓGICO MERLEAU- PONTYANO, MÁS ALLÁ DEL ESPACIO EUCLIDIANO Y DEL PENSAMIENTO REFLEXIVO}

En este artículo nos proponemos interrogar los distintos modos del cuerpo que se vislumbran en el campo narcisista y en el registro pulsional. Partimos de algunos desarrollos de los últimos años de Merleau-Ponty, en la búsqueda de fundamentar mejor esta diferencia. Para ello, pondremos el acento en las vicisitudes de la inserción del cuerpo en el mundo, en relación con lo que este filósofo ha promovido bajo el nombre de carne.

Pese a la afirmación de Lacan que sitúa los límites de la fenomenología merleau-pontyana en la posibilidad de dar cuenta del fantasma, ${ }^{1}$ buscaremos mostrar cómo, en lo referido a la espacialidad y la temporalidad del cuerpo, ella nos instala de lleno en una configuración de lo corporal que excede en psicoanálisis el ámbito del narcisismo especular, en contra de lo que podría hacer pensar el nombre de narcisismo de la visión, escogido por el fenomenólogo para los modos de la Visibilidad que buscar cernir (Merleau-Ponty, 964: 181).

Los abordajes de Merleau-Ponty en Lo visible y lo invisible nos fuerzan a desprendernos del espacio euclidiano, para suplantarlo por un espacio topológico presentado como el verdadero "mode- lo del ser". El primero, calificado como un espacio que no tiene trascendencia, es connatural al "pensamiento reflexivo" (Merleau-Ponty, 1964: 260), mientras que el segundo permite, en cambio, confrontar a éste contra un resto irreductible que no puede captar. ${ }^{2}$

Para dar cuenta de la presencia en el espacio es preciso rechazar la idea de una "conciencia ante un noema". Existen una serie de diferentes niveles que permiten exponer las características del espacio topológico, enmarcadas tanto en el cuestionamiento de la "idealización cartesiana" como en la objeción de la concepción husserliana del "flujo de Erlebnisse individuales" (Merleau-Ponty, 1964: 289).

Merleau-Ponty expone una serie de figuras que permiten pensar la relación del cuerpo con el mundo enmarcada en este espacio topológico. El punto de partida para definirlo puede pesquisarse en el "torbellino espacializador-temporalizador", que rechaza las ideas de ego y conciencia como centros de la experiencia. Esto conduce a una figura subsiguiente, que implica un "enroscamiento sobre sí" (Duportail, 2011: 81-82), y que da lugar a una torsión, posible por ese atributo fundamental de la carne, la flexibilidad, que funciona al modo de un "tejido o una superficie de goma". Luego se produce un pliegue, que "instituye una distancia, un hueco en el tejido general del ser visible”, y que funciona como un punto de vuelta en que el revés hace retorcer sobre sí mismo al campo total del ser, engendrando así "por torsión la diferencia entre el cuerpo vidente y el cuerpo visible” (Duportail, 2011: 83).

Por una parte, es posible situar un contrapunto entre los espacios que Merleau-Ponty y Lacan configuran: el punto de vuelta es un pliegue y no un vacío, 
"es una torsión y no un corte" (Duportail, 2011: 88), lo que no bastaría según este autor, para precisar la dimensión del objeto perdido del psicoanálisis. No obstante, aunque el corte no sea una figura explícita en Lo visible y lo invisible, es posible deducirla de sus desarrollos. Que la torsión no sea necesariamente excluyente de la operación del corte conduce a Duportail a sugerir que el "investimiento de un ente" podría oficiar como algo homólogo de la función del objeto en el fantasma, pues incluiría a su modo "el corte del sujeto en un objeto perdido” (2011: 90-91). Aunque resulte de interés dejar precisada esta cuestión, no es allí, sin embargo, que pondremos el acento. Interrogaremos la pertinencia de la función del corte, pero lo haremos en otros aspectos de la corporeidad que Merleau-Ponty instituye en relación con la carne, lo que nos permitirá avanzar en la problematización del estatuto del cuerpo pulsional.

\section{REVERSIBILIDAD E IRREVERSIBILI- DAD DEL NARCISISMO DE LA CARNE: EL MUNDO Y EL CUERPO}

Con la afirmación de que la "actividad es idénticamente pasividad” (1964: 181) Merleau-Ponty postula un narcisismo inherente a la Visibilidad, que implica una contracara pasiva para toda visión ejercida activamente. A partir del intercambio recíproco entre vidente y visible no es posible establecer qué es lo que ve y qué es lo visto.

Este narcisismo anclado en la noción de carne apunta a atenuar en cierta manera el poder nadificante que el hombre ejercería sobre el mundo, contrarrestando su actividad por una pasividad que le es aún más constitutiva. La idea inicial que
Merleau-Ponty formula, que la carne del mundo no podría explicarse por la carne del cuerpo puesto que es la primera la que permite "comprender el cuerpo propio" (1964:299), se matiza cuando tomamos en cuenta la simultaneidad de tres fenómenos que en otras formulaciones expone: "La carne del mundo no es explicada por la carne del cuerpo o ésta por la negatividad o el sí que la habita"(1964: 298, destacado nuestro).

Si destacamos esto es porque permite ver que la espacialidad y la temporalidad que Merleau-Ponty propone para el cuerpo están dadas por una negatividad muy particular: la que se establece en la relación del cuerpo consigo mismo; como si esa negatividad, atenuada en sus efectos corrosivos sobre la carne del mundo (¿trasfondo de reproche a la acción negativa de una conciencia que corroe?), quedara desplazada a un sector muy específico, que aquí nos resulta de interés para entender un modo no narcisista del cuerpo (ahora en el sentido que esta noción cobra en psicoanálisis).

Ese sí del que habla Merleau-Ponty, limitado en sus alcances sobre el mundo, parece quedar restringido al movimiento del cuerpo sobre sí mismo. Es decir que lo que desde cierta perspectiva pareciera negar rotundamente, la existencia de un corte entre el cuerpo y el mundo - "Las cosas [...] son un anexo o una prolongación de él mismo, están incrustadas en su carne, forman parte de su definición plena y el mundo está hecho de la estofa misma del cuerpo"aparece de golpe revelado en esa diferencia que deja sedimentada la carne: la reversibilidad que potencialmente se produciría en el mundo -y que en los términos de "El ojo y el espíritu" puede ser vinculada a la "indivisión de lo sentiente y lo sentido" (Merleau-Ponty, 1961: 197)- es interrum- 
pida en el plano del cuerpo, porque en el intento de consumar un movimiento sobre sí algo se escapa.

Narcisismo es de hecho el nombre que Merleau-Ponty da a esa inherencia de la actividad a la pasividad, lo que permite cifrar un mínimo punto de contacto con la noción de narcisismo en psicoanálisis — aunque en todo lo demás difiera-, pues si hay una polaridad que la reciprocidad narcisista compromete, ella es precisamente la de la distinción entre actividad y pasividad, como más abajo lo veremos.

Ahora bien, a esa indivisión entre actividad y pasividad que caracteriza a la carne del mundo, Merleau-Ponty la hace colapsar en el plano del cuerpo propio, mostrándonos que no hay reversibilidad del sí mismo (para evitar confusiones es importante notar que cuando Merleau-Ponty habla de narcisismo no está situándose en el plano de la relación que el cuerpo guarda consigo mismo ni con sus semejantes; lo que nombra narcisismo es más bien cierta configuración que cobra la presencia del ser corporal en el mundo). A partir de esto quisiéramos apuntar que, al ubicar las consideraciones respecto de un cuerpo que efectúa un movimiento sobre sí, en lugar de trasladarnos al campo del narcisismo especular, nos propicia una apertura al terreno psicoanalítico del cuerpo pulsional.

Lo importante a destacar es que en la tentativa del existente corporal por coincidir reversiblemente consigo mismo, buscando vivir la contracara pasiva de la actividad que ejerce, y consumar en ese acto la coincidencia de lo activo y lo pasivo en una actualidad corporal, algo se pierde: "lo mismo en mi es visto y vidente (voyant). No me veo ni siquiera viendo (voyant), mas por desbordamiento (empiètement) termino mi cuerpo visible, prolon- go mi ser visto más allá de mi ser visible para mí" (Merleau-Ponty, 1961: 253). Esa visibilidad propia que al cuerpo se le escapa pareciera quedar plasmada o efectuada en el plano del mundo. El producto de esa sustracción en el cuerpo no deja de formar parte de una carne del mundo que viene en cierto modo a incrementarse, lo que permite acentuar la discrepancia de los dos niveles (cuerpo y mundo): "esta laguna en que se encuentran nuestros ojos es colmada, colmada por algo aún más visible, pero del que no somos titulares" (Merleau-Ponty, 1961: 186, destacado nuestro).

Lo que se impone a partir de esta consideración es que esa falta de titularidad en la visibilidad del propio cuerpo parece no corroer al mundo, que no ve reducida en nada su potencial visibilidad; lo que queda alterado en ese movimiento es la mismidad del cuerpo. Es este defecto que se produce en el cuerpo lo que permite pensar ciertas similitudes con el cuerpo afectado en psicoanálisis por un objeto caduco.

A la operatoria del objeto la encontramos en la resta que puede leerse entre la carne del mundo y la carne del cuerpo, y no por el contrario en el modelo topológico de una reversibilidad consumada, como parece creerlo Bernard Baas (2011) ${ }^{3}$ cuando analiza los parentescos entre los modos del espacio propuestos por Merleau-Ponty y Lacan. Intentaremos mostrar que no es el acento en la supuesta reversibilidad del cuerpo, tal como este autor lo propone, lo que nos acerca a la tramitación pulsional de lo corporal en el eje del fantasma.

Para dilucidar estas cuestiones desde el campo analítico es de extrema importancia abordar el modo de tratamiento del objeto. Por ello nos enfocaremos en cuestionar la idea que promueve Baas al hacer coincidir el objeto a con la reversibilidad teorizada 
por Merleau-Ponty (2011: 107). Consideramos que plantear esa coincidencia nos impide pensar el cuerpo pulsional en su negatividad, a la vez que aplasta la riqueza escópica de lo corporal en el espacio merleau-pontyano. Si encontramos una suerte de réplica de la función del objeto a como resto en la carne del cuerpo, es precisamente, como lo venimos apuntando, en el punto en que impone como manifestación de su negatividad un límite a la reversibilidad.

Tal como es concebida por Lacan en sus primeros abordajes, la constitución especular narcisista produce como efecto fundamental la ilusión de reciprocidad entre el vidente y su imagen en el plano de la constitución del yo (Carignano, 2017). Esa reciprocidad es sin resto. Merleau-Ponty al poner en primer plano el resto que corroe todo movimiento del cuerpo sobre si mismo, nos abre otra dimensión de lo corporal (que aquí buscaremos vincular con el cuerpo que se inscribe en el registro de lo pulsional anclado en el fantasma).

\section{Paréntesis Sobre el CUerpo SUbli- MADO: MODO DEL CUERPO PULSIONAL SUSTRAÍDO DE LA NEGATIVIDAD}

Por la vía del arte pictórico, poniendo en escena el cuerpo sublimado, Merleau-Ponty da cuenta de las particularidades de un modo de trabajo de lo pulsional que pareciera sustraerse a la corrosión de la negatividad. Cuando interroga a ese cuerpo que la pintura produce, nos abre a otra figura de la carne del cuerpo que no debe despistarnos (puesto que excede el límite que funciona en la carne del cuerpo).

En “El ojo y el espíritu” se nos deja así entrever que lo que podríamos apuntar como destino sublimatorio de lo pulsional conduce a la disolución del límite que la corporeidad propia impone a la carne del mundo.

Esta cuestión se enmarca en la distinción que es preciso efectuar analíticamente entre el cuerpo trabajado por el destino sublimatorio y el cuerpo sometido a la economía de la pulsión parcial anclada en el fantasma, asociada a un objeto caduco en función de causa.

$\mathrm{Al}$ cuerpo de la sublimación lo podemos leer en Merleau-Ponty, en la expresión de la reversibilidad entre lo vidente y lo visible. Es el cuerpo que conduce a la pérdida de referencia respecto de "quien ve y quien es visto, quien pinta y quien es pintado", el que produce la verdadera "inspiración y expiración del Ser” (1961: 202), mostrando en un quiasmo universal la pertenencia sin límites del cuerpo al mundo. (Veremos más adelante cómo la ontología de la carne deducida de la fantasmática kleiniana se parece más a esa figura del cuerpo sublimado que a una expresión de la articulación cuerpo-fantasma tal como podemos pensarla a partir de la irreversibilidad de la pulsión).

Cuando Merleau-Ponty se dirige desde allí a examinar la reflexividad de lo sensible en el espejo deja manifiesto un desnivel que muestra una fractura con ese cuerpo de lo especular. En disrupción con el paso previo por el sí mismo nos expone esa figura de reflexión sobre sí que el pintor ejecuta pintándose, donde puede leerse un movimiento pulsional sublimado que desvía la economía narcisista de lo corporal.

Merleau-Ponty parte de la invisibilidad de mi cuerpo como condición de investimiento de otros cuerpos que veo. En lo que esboza, puede leerse entre líneas la identificación imaginaria del ego: "el hombre es espejo para el hombre” (1961: 203). Sin 
embargo, a la hora de situar el cuerpo se nos revela el desnivel con este registro del cuerpo en relación con el semejante; lo vemos cuando unas líneas más abajo busca figurar la cuestión a partir de ese gusto singular del pintor por pintarse pintando. Queda claro en su planteo que el pintor no busca en modo alguno una imagen de sí mismo, sino que se dirige a la disolución del sí mismo que le permite la pintura en tanto que es agencia de una inspiración y expiración universal del Ser: al dejar plasmado en la tela lo que "las cosas veían de ellos", los pintores no hacían otra cosa que "demostrar que hay una visión total o absoluta, fuera de la cual nada permanece, y que se cierra sobre ellos mismos" (1961: 203).

El arte del pintor es lo que parece manifestar de forma más pronunciada a la carne como "anonimato innato de Mí-mismo" (Merleau-Ponty, 1964: 181), es decir, es lo que expone mejor que nada el punto en que la distinción entre la carne del mundo y la del cuerpo se encamina a un eclipse. No es pues en este cuerpo sublimado, que dedujimos de la experiencia del pintor comentada por Merleau-Ponty, que puede vislumbrarse la negatividad que corroe al cuerpo, pues esta última exige que la distinción entre carne del cuerpo y carne del mundo se mantenga en pié.

\section{LA CARNE DEL CUERPO Y LA CARNE DEL MUNDO}

Aunque sea con el nombre de narcisismo que Merleau-Ponty busca sellar su quiasmo entre actividad y pasividad, ese narcisismo nada tiene que ver con el que el psicoanálisis circunscribe a partir del imaginario especular. Lo que el narcisismo especular viene a obturar, es decir, lo que se pierde en todo movimiento del cuerpo sobre sí, es por el contrario revelado en el narcisismo de la carne a partir de la reversibilidad fallida del cuerpo.

En pos de precisar lo que hemos expuesto, es necesario distinguir en la carne los dos niveles siguientes:

1) En primer lugar, la carne del mundo, donde la reversibilidad se vislumbra en un horizonte de consumación, en que la negatividad es confrontada con un reverso que pareciera positivizarla, de lo que podría ser expresión la síntesis de un quiasmo universal. La reversibilidad de la carne se figura en esa "dehiscencia del vidente en visible y del visible en vidente" (Merleau-Ponty, 1964: 199), suerte de desdoblamiento perpetuo que asegura un quiasmo entre actividad y pasividad en ese hecho primario que es la "visibilidad anónima" de un mundo que no tiene autor (1964: 197). La figura de todos lo quiasmos pudiendo ser albergados por un único quiasmo - "los quiasmos múltiples no constituyen más que uno solo” (Merleau-Ponty, 1964: 309)- es reveladora de la concepción de la carne del mundo como indivisión del Ser sensible que soy" (1964: 303). Esa indivisión, expuesta mejor que en ningún otro caso en el cuerpo sublimado del pintor (como más arriba lo vimos), es la de un cuerpo que no está constituido en el horizonte de una sustracción que opera como resto.

2) Esa indivisión general de la carne del mundo se encuentra con una especie de límite interno: la carne del cuerpo. Aquí es donde apuntamos el segundo nivel de- 
cisivo, que manifiesta el impedimento del cuerpo en cerrarse sobre sí, en coincidir consigo mismo (ilusión que en otro registro permite sostener al narcisismo especular como lo que oblitera todo rastro de pérdida). Si el término división podría objetarse como abusivo e inapropiado, la noción de bougé, tomada del vocabulario fenomenológico merleau-pontyano, permite igualmente precisar la idea de una dislocación próxima en sus efectos a los que resultarían del corte. El efecto de corte puede situarse en ese núcleo que caracteriza a la sensibilidad corporal del sentiente, en el punto en que algo se pierde del ser sensible que es.

"La relación de mi cuerpo como sensible con mi cuerpo como sentiente (el cuerpo que toco, el cuerpo que toca) = inmersión del ser tocado en el ser tocante y del ser tocante en el ser tocado" (Merleau-Ponty, 1964: 308). Mi ser visible puede acabar produciéndose pero estoy impedido de captarlo, hay un desfase entre actividad y pasividad fundamentado con la temporalidad de una inminencia. La transformación siempre posible de lo activo en lo pasivo, y viceversa, muestra en el plano del narcisismo de la carne del cuerpo un carácter fundamental de ausencia de consumación: "[...] mi mano izquierda está siempre a punto de tocar mi mano derecha tocando las cosas, pero nunca llego a la coincidencia; ella se eclipsa en el momento de producirse [...]" (1964: 191). En la falta de coincidencia "[...] no se trata de un fracaso, si escapan al momento de coincidir, si hay siempre entre ellas algo de 'movido' (bougé), un 'distanciamiento', es precisamente porque mis dos manos forman parte del mismo cuerpo [...]" (1964: 192, destacado nuestro).
Lo que interrumpe la reversibilidad es la relación del cuerpo consigo mismo. La negatividad que lo corroe hace posible pensar el registro de la irreversibilidad que constituye al cuerpo como pulsional (en contraposición al narcisismo y a la economía autoerótica de satisfacción).

El narcisismo supone por el contrario una elisión de la fractura del cuerpo. La alteridad queda velada precisamente por la inexistencia de un resto que la manifieste: "por la forma $i(a)$, mi imagen, mi presencia en el Otro, es sin resto" (Lacan, [1962-63], 2004: 292). La obturación de esa falta que se inscribiría a modo de resto es la condición del sostenimiento narcisista en el campo del Otro. Esto implica, como lo veremos a continuación, que el objeto propio de la libido narcisista no pueda en modo alguno pensarse como funcionando en la economía del resto. (Esto ha sido clásicamente desconocido en muchas ocasiones, aún por autores que usan el ropaje del lenguaje lacaniano, lo que ha llevado al aplastamiento entre los niveles de la pulsión parcial y del narcisismo ${ }^{4}$ ).

La no coincidencia del cuerpo consigo mismo manifiesta la apertura a una alteridad que el narcisismo especular está destinado a ocultar. Esto permite pensar que la carne del cuerpo, en tanto impone un limite a la reversibilidad de la ontología de la carne del mundo, es una expresión del trabajo de lo pulsional en el cuerpo.

Tomando en cuenta lo hasta aquí expuesto, buscaremos avanzar en la distinción entre la carne del cuerpo pulsional y el cuerpo narcisista. Para esto examinaremos, por un lado, cómo funciona lo pulsional del cuerpo en el registro autoerótico, punto en que se suele situar el fundamento de una similitud cuestionable entre los espacios que esbozan Merleau-Ponty y La- 
can. Por otro lado, interrogaremos el cuerpo que la dialéctica kleiniana inscribe en su particular concepción de las fantasías, puesto que es en él que el fenomenólogo ha creído encontrar un modelo del psiquismo que se aproxima a los supuestos de su propia ontología de la carne. Pese a la apoyatura de Merleau-Ponty en los desarrollos teóricos de Klein para fundamentar lo que llama una ontología de la carne, trataremos de mostrar por qué, en sus consideraciones derivadas, parece quedar soslayada la distinción clave entre la carne del cuerpo y la carne del mundo, aquella desde la cual podemos vislumbrar la negatividad del cuerpo pulsional en psicoanálisis.

\section{LA RECIPROCIDAD DEL NARCISISMO Y LA} TRASCENDENCIA DEL OBJETO EN EL CUERPO PULSIONAL

La ilusión propia del imaginario narcisista se ancla en la creencia de una reciprocidad entre la acción de ver homologada al pensamiento (la conciencia enmascarada en el $\operatorname{cogito}^{5}$ ) y lo que se ve en la propia imagen especular, coartada para situar el trabajo de lo pulsional en el cuerpo.

Este engaño de reciprocidad del imaginario especular se funda en la proclama de que en el acto de ver puedo verme viendo, es decir, de que habría coincidencia entre el lugar en que me constituyo como vidente y el espacio en que soy visible. Ello produce ese ocultamiento de la incidencia pulsional en lo escópico pesquisada por Lacan como esquizia entre visión y mirada (ampliándola a generalidad de lo corporal, podríamos también vincularla con el desfase entre lo tocante y lo tocado del que se ha ocupado Merleau-Ponty).
A la reciprocidad es preciso inscribirla en el orden del narcisismo, mientras que la pulsión introduce, enlazada al fantasma, un modo del objeto en que puede pesquisarse una alteridad radical, irreductible a la constitución narcisista (del yo y de los objetos):
Afirmo la distinción radical que existe entre el amarse a través del otro -cosa que no permite, en el campo narcisis- ta del objeto, ninguna trascendencia al objeto incluido en él-y la circulari- dad de la pulsión, en la que la hetero- geneidad entre la ida y la vuelta reve- la una hiancia en el intervalo (Lacan, [1964c], 1987: 201).

La dimensión del gran Otro es alcanzada en el movimiento que supone contornear el objeto $a$, dimensión que permanece estructuralmente eclipsada en el campo narcisista del amor. Una alteridad fagocitada es el resultado del funcionamiento mismo del narcisismo, nivel nuclear que instaura una reciprocidad ilusoria entre el yo y el semejante, en que se oculta todo rastro de pérdida estructural del objeto.

La dimensión de pérdida significante permanece obliterada en este plano, mientras que, en el nivel pulsional, el objeto $a$ (la mirada para el caso escópico), se presenta como "objeto intragable", un resto "que permanece atravesado en la garganta del significante" (Lacan, 1964b: 434). A diferencia de lo que ocurre en el plano imaginario en que se inscribe el objeto narcisista, en el caso de la pulsión nos encontramos con un objeto transcendente que implica "fundamentalmente actividad", transcendencia borrada, obliterada como tal en el registro narcisista. "[...] A nivel del amor hay reciprocidad entre amar y ser 
amado, mientras que en el otro campo [el de la pulsión] sólo se trata de una pura actividad" (Lacan, [1964c], 1987: 208).

El modo de relación con el Otro a través del objeto diverge en ambos planos, lo que determina una constitución diferente del cuerpo, que más arriba pudimos vislumbrar al tomar en cuenta la espacialidad y la temporalidad de la carne.

La distinción entre la dualidad recíproca del narcisismo y el tiempo fantasmático de la pulsión (el tercer tiempo en el esquema freudiano que Lacan retoma), permite iluminar mejor la ausencia de reciprocidad en el nivel fantasmático. Mientras que el imaginario narcisista supone un modo del cuerpo desembarazado de la pulsión, el que es propio del fantasma encuentra en ésta un elemento que le es constitutivo y que interviene alterando la estofa narcisista que constituye su trasfondo (el del fantasma).

El fantasma lleva incrustado en su estructura la trascendencia del objeto de la pulsión. Este movimiento es fundamental para mostrar por qué la dimensión psíquica del fantasma no supone una introyección empírica de objetos que funcionaría como réplica de una parte de la realidad. Por tanto, el fantasma no puede pensarse como una incorporación reversible del mundo donde quedaría borrada la diferencia entre interioridad del cuerpo y exterioridad del mundo, en una especie de quiasmo universal (que es la figura fantasmática preconizada por Merleau-Ponty al promover una ontología kleiniana de la carne).

Para precisar el estatuto del objeto del fantasma es ante todo fundamental situar su ruptura con la economía del autoerotismo (a este respecto podríamos proponer la expresión "autoerotismo del objeto" como un oxímoron sugestivo de la imposibilidad de pensar la transcendencia del objeto fantasmático en continuidad con la economía autoerótica de satisfacción). Un trasfondo psíquico de narcisismo, que incorpora en un segundo movimiento un objeto extraño a su economía, es fundamental para dar cuenta de la estructura del fantasma. El objeto $a$, en su carácter trascendente, se incrusta como una alteración en esa reversibilidad narcisista de base. (En contraposición a ello, como lo veremos más abajo, en la ontología de la carne descubierta por Merleau-Ponty en Klein la reversibilidad parece funcionar anulando el valor trascendente y propiamente fantasmático del objeto).

Por inscribirse en el registro de un campo escópico que excede las coordenadas del imaginario especular, la mirada es de los diversos objetos el que mejor deja expuesto el excedente al campo narcisista. En su carácter de resto, este objeto permite ubicar el refugio de un "goce que no entra en el dominio del principio del placer". Al no ser captada como un "reflejo del cuerpo", la mirada puede ser postulada como un resto del reflejo del cuerpo que excede la economía de la "estesia representativa reguladora del principio del placer $[\ldots]$ estesia representativa donde el individuo se encuentra y se apoya, identificado a sí mismo, en la relación narcisista en que se afirma como individuo". (Lacan, 1966-67: 520). El objeto caído del cuerpo del que dispone el fantasma no está al servicio de una economía de reciprocidad en la relación del sujeto con el Otro, tal como las estructuraciones voyeurista y exhibicionista lo hacen palpable. Ellas dejan en claro desde otro ángulo el desfase que impide inscribir al masoquismo y al sadismo como figuras recíprocas. 
VI . LA NO TRASCENDENCIA DEL OBJETO EN EL CUERPO NARCISISTA DEL YO-PLACER Y LA INSTAURACIÓN PARADÓJICA DEL OBJETO DEL FANTASMA (OBJETO MALO INTERNO)

Sobre el final del seminario Los cuatros conceptos..., Lacan vuelve a echar otra luz a la incompatibilidad de registros entre narcisismo y pulsión retomando el LustIch (Yo-placer) freudiano a partir del siguiente esquema (Lacan, [1964a], 1973: 267):

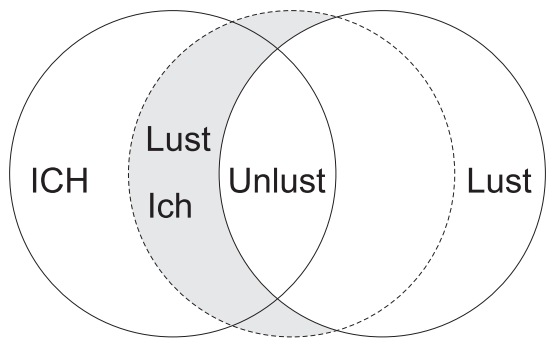

El yo se define por estar acoplado a un funcionamiento bomeostático del aparato psíquico (Lacan, [1964a], 1973: 266). Si hay articulación entre homeostasis y placer es porque la primera supone una rebaja óptima de energía que el principio del placer tendería a asegurar, donde placer equivaldría, de acuerdo a la temprana formulación freudiana de ese principio, a rebaja de excitación en el aparato. De allí se deriva un uso particular del término autoerotismo que la lectura de Lacan permite desbrozar, aunque ya esté de todos modos presente en el ensayo de Freud sobre las pulsiones ([1915], 1986).

Dicho uso está en consonancia con la constitución del yo y su modo de amarse en objetos sin trascendencia, pues esa es la regla misma de la constitución del Yo-pla- cer, que luego subsiste en la vinculación erótica del yo con sus posteriores objetos de amor. Si el autoerotismo supone "[...] no la inexistencia de los objetos, sino el funcionamiento de los objetos únicamente en relación con el placer" (Lacan, [1964a], 1973: 267), también sirve para calificar ese momento de constitución del yo en que éste coincide con placer. Este modo de vinculación con los objetos es homologable, en cierto punto, al de la pulsión autoerótica con su objeto eclipsado. De allí que Freud pueda retener la noción de autoerotismo para nombrar un modo de satisfacción que opera en un nivel diferente, el narcisista ([1915], 1986). En el caso del Yo-placer, el alcance del término autoerotismo se restringe a la idea de que que no hay otros objetos amados diferentes del yo, siendo el yo el único objeto de placer.

El esquema propuesto por Lacan figura en la intersección de dos círculos, el del Ich (yo) y el del Lust (placer), el espacio para el Unlust (no placer), irreductible a ambos. El agregado del tercer círculo en líneas de puntos sirve para delimitar el espacio en que Lust e Ich coinciden formando el Lust-Ich (Yo-placer). El Lust es un objeto de placer "mirado en el yo (moi)" (Lacan, [1964a], 1973: 266, destacado nuestro). Que sea mirado en el yo quiere decir que no posee alteridad respecto del yo, no conecta al yo con algo otro de sí mismo.

Aquí, en la génesis del yo articulado al placer, momento originario del surgimiento de un objeto amado que no es otro que yo, está el fundamento, bajo la forma del primer narcisismo, de la reciprocidad como modo que caracteriza cierto tipo de vínculo con los objetos. ${ }^{6}$

La atracción que el objeto amado despierta, y que Freud interpreta con la preci- 
sión de que se trata de aproximarlo lo más posible al yo, mostrando con evidencia los orígenes psíquicos del amor, es un relicto de esta etapa originaria del amor ([1915], 1986). Amar al objeto quiere decir atraerlo hacia el yo, que éste quiera en cierta medida incorporárselo, eliminando su otredad para fundirse con él; en los términos de Lacan, mirar al objeto en el yo implica abolir su trascendencia.

La pulsión se aloja, por el contrario, atópicamente en el campo del Unlust, del no placer, de lo irreductible a la economía homeostática del Yo-placer. En el Unlust se instituye el no-yo, que muerde sobre el círculo del yo primitivo, “[...] sin que el funcionamiento homeostático llegue nunca a reabsorberlo"; punto de localización de lo que se ha promovido en la teoría analítica como objeto malo (Lacan, [1964a], 1973: 268).

La disyunción entre narcisismo y pulsión es fundamental para indicar el carácter atópico del fantasma en el entrecruce. A la paradoja que supone la concepción del objeto malo interno, Lacan otorgaba ya, en su sexto seminario, un papel clave en la creación del lugar psíquico del fantasma (1958-59). Dicho objeto instituye algo separado en el seno mismo del psiquismo, un aparte no tópico, inscripto en otro plano, y que se relaciona con el resto de los representantes como un desgarramiento de su trama. El objeto del fantasma, resultado de la inserción del objeto pulsional trascendente en el seno del campo regido por la libido narcisista, es así instituido como algo extranjero y a su vez interno a esa economía imaginaria del yo-corporal.
VII. EL ABRAZO CARNAL DEL AUTOEROTISMO COMO MODELO TRUNCO DEL CUERPO PULSIONAL

El objeto de la pulsión trasciende el plano dual y recíproco del objeto narcisista. El origen de lo pulsional no es el propio cuerpo, la causa no está subsumida en la fuente corporal. La pulsión opera como extraída del cuerpo en un trayecto de vaivén que la hace retornar luego, pero dejando siempre abierta una hiancia, la ranura registrada en el cuerpo por la tramitación de la pérdida que funciona como causa.

Esto también había sido precisado por Lacan con un esquema anterior del mismo seminario (Lacan, [1964a], 1973: 200), acompañado de la siguiente formulación: "Algo que sale de un borde, que redobla la estructura cerrada, siguiendo un trayecto que retorna, y del que ninguna otra cosa asegura su consistencia más que el objeto, a título de algo que debe ser contorneado" ([1964a], 1973: 203).

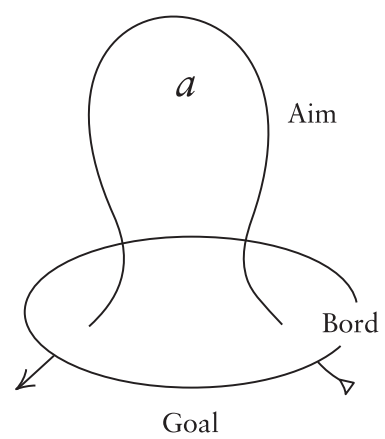

La metáfora freudiana de la boca que se besa a sí misma, "boca flechada" o "boca cosida" (Lacan, [1964a], 1973: 201) supone, en realidad, un modelo trunco de la 
pulsión. Podríamos decir que no hay allí fantasma en juego sino mero autoerotismo.

Todo uso del término autoerotismo en relación con el fantasma es cuestionable porque éste no se presta a ningún funcionamiento auto de lo erótico.

La actividad pulsional en su anclaje fantasmático (tercer tiempo freudiano), da la medida de la distancia con esa versión de lo autoerótico que opera en el plano del amor narcisista (tramado en la economía autoerótica del yo, por más objeto exterior que pueda encontrarse invistiendo). Por esto es que consideramos preferible reservar el término autoerotismo para los déficit en la constitución del fantasma, y circunscribir el erotismo del cuerpo acoplado al fantasma con la noción freudiana de placer de órgano (Freud, [1915], 1986: 121). Dicho placer es un resto del autoerotismo, pero puesto a funcionar en otro registro psíquico.

Aunque no establezca una distancia conceptual del todo precisa, Lacan esboza una diferenciación terminológica de importancia al respecto: el autoerotismo de la boca cosida, manifiesto en esos silencios en que se expresa "la instancia pura de la pulsión oral, cerrándose sobre su satisfacción", debe ser distinguido "del puro y simple autoerotismo de la zona erógena" (Lacan, [1964a], 1973: 201).

Si bien Freud caracteriza explícitamente como narcisista al segundo tiempo de la pulsión, creemos que de ello no se debe concluir que la versión pasiva de la pulsión responde al narcisismo, como algunos abordajes de la metapsicología de la pulsión de ver lo suponen. ${ }^{7}$ Ello conduce al alejamiento de algunos supuestos fundamentales para distinguir los órdenes sobre cuya heterogeneidad aquí insistimos.
Los desarrollos de las últimas páginas de "Pulsiones y destinos de pulsión" son cruciales para lo que buscamos aquí proponer (Freud, [1915], 1986). Esa densa parte del texto expone argumentos que Lacan permite iluminar de forma ejemplar, según lo acabamos de ver, mostrando la disparidad de los dos órdenes pulsional y narcisista en función del modo de tratamiento de la alteridad del objeto.

En el plano del narcisismo no hay resto, el objeto de amor, o el yo mismo como objeto, están allí para taponar la falta. Como más arriba lo precisamos, la imagen especular narcisista se inscribe en el campo del Otro sin dejar ningún resto (Lacan, [1962-63], 2004: 292). No hay pues, desde la economía del narcisismo, percepción posible de la falta en el Otro.

$\mathrm{Al}$ contrario, el campo pulsional y fantasmático suponen que el resto funcione en tanto tal, en su dimensión de pérdida: para que haya un deseo ligado (attaché) a la imagen es necesario circunscribir un "[...] corte que sobreviene en el ojo" ([1962-63], 2004: 265). El corte produce un resto, la mirada como objeto perdido del cuerpo; corte en el ojo que se extiende evidentemente a la imagen, ¿de qué otro modo podría sino tornarse materialidad para el deseo?

Los planteos de Baas nos parecen desacertados en este punto. Este autor plantea a la reversibilidad de la pulsión como "una adherencia que hace que se confundan el interior y el exterior" (Baas, 2011: 116), cuando, tal como lo hemos visto, el movimiento de la pulsion supone todo lo contrario. Si los labios que se besan a sí mismos no dan cuenta del funcionamiento propio de la pulsión es porque en realidad no hay tal reversibilidad consumada en el plano pulsional (que llevaría a la confu- 
sión entre interior y exterior). Esa figura de los labios constituye más bien el limite autoerótico —real- de la pulsión y no el modelo típico de una pulsión parcial articulada al fantasma:

La relación con la alteridad es un contacto entre el reverso y el anverso, un abrazo carnal de la superficie general del mundo auto-desdoblado, al modo de los labios que se besan a sí mismos. El cuerpo y el mundo, provenientes de una misma carne, se abrazan [s'embrassent, se besan] uno a otro (Duportail, 2011: 84).

La boca que se besa a sí misma sirve para fundamentar la reversibilidad carnal consumada (y es por ello mismo que no puede oficiar como modelo para caracterizar a la pulsión parcial articulada a un objeto resto en función de causa). No se trataría propiamente de alteridad, según esta caracterización que establece Duportail respecto del contacto entre anverso y reverso, sino más bien de un movimiento que tiende a su disolución.

Asimilar la corporeidad pulsional a la reciprocidad o la reversibilidad de Merleau-Ponty, entendidas como una lógica "de lo mismo que se da vuelta" (Baas, 2011: 112), lleva a desconocer la especificidad del cuerpo atravesado por la pérdida del objeto. El modelo del autoerotismo que acabamos de examinar es lo que extravía a este autor a la hora de dar cuenta del estatuto de la pulsión parcial. La asimilación que propone Baas entre el objeto $a$ y la reversibilidad merleau-pontyana (2011: 107), no tiene otro efecto que el de anular el estatuto del objeto en su dimensión de resto. Al estatuto del objeto es preciso ubicarlo en otro lugar: en la figuración del trayecto pulsional que expresa más bien la hiancia que queda en el mo- vimiento de retorno, lo que pudimos más arriba articular con la carne del cuerpo, en la medida en que registra el fracaso en la consumación del movimiento reversible.

La reversibilidad malograda, fallida, que "no es identidad actual de lo tocante y lo tocado" (Merleau-Ponty, 1964: 320), y cuyo eje se ubica en los modos de relación del cuerpo consigo mismo es lo que permite dar cuenta del cuerpo pulsional. Allí es donde se revela una fragmentación contraria al funcionamiento de una totalidad unificada.

Lo que interesa no es, pues, la vinculación del cuerpo consigo mismo, sino las vías por la cuales el fracaso de la totalidad corporal (ligada a la ausencia de cierre sobre sí) permite, en el horizonte de lo fragmentario del cuerpo, desplazar el problema a las vicisitudes locales de las pulsiones parciales en relación con sus propias fuentes. El cuerpo que fracasa en la reversibilidad es el que revela los defectos que le impiden funcionar como totalidad unificada, es decir, el cuerpo atravesado por la parcialidad de los movimientos locales de las pulsiones.

Era de esperar que el precio de no haber precisado el estatuto del objeto como resto en la economía de la pulsión parcial se replique en las consideraciones sobre el fantasma: al afirmar que la función del $l o-$ sange en la fórmula del fantasma es la de indicar la reversibilidad (2011: 119), Baas no puede leer la paradoja inclusión-exclusión como modo de incorporación de los objetos fantasmáticos a un interior psíquico paradójico (que no constituye propiamente ningún interior, como lo quisiera el espacio psíquico kleiniano de las fantasías). Esto queda claramente explicitado en la recuperación que Lacan efectúa del 
objeto malo interno como instituyendo el espacio paradójico del fantasma, tal como más arriba lo vimos.

Estrictamente, la formulación "hacerse amar" no sería apropiada para dar cuenta de un destino decisivo para el objeto de amor, cuando se lo liga a lo "propiamente narcisista” (Assoun, 2001: 46). La desventaja de esa formulación es la de evocar la estructura del tercer tiempo de la pulsión para inscribir allí al amor. Los efectos indeseables de esa inscripción no serían otros que los consiguientes: 1) Anular la alteridad radical que supone el hacerse de la pulsión en el tercer tiempo, donde el objeto subsiste como un resto irreductible al cuerpo; 2) Hacer del amor narcisista algo no recíproco.

En contraste con ello, si hay verdaderamente una formulación que permitiría circunscribir la reciprocidad del narcisismo, es la que Freud expone en relación con el segundo tiempo de la pulsión, que en realidad pareciera responder más a las coordenadas narcisistas que a las pulsionales; su formulación es la del reflexivo simple: amarme, centro nodal de las versiones activa y pasiva: amar y ser amado. El hacerse del tercer tiempo, no presente como tal en Freud, y producto de una lectura de Lacan, permite demostrar, suplantando el werden del texto freudiano por el verbo machen, la alteridad radical que supone el objeto en una pulsión esencialmente activa, aunque pueda tener meta pasiva; o sea, de ningún modo recíproca (es decir, de una suerte de reversibilidad lograda que anularía el papel trascendente del objeto).

El hecho de que los tiempos de la pulsión no se inscriban en una reversibilidad simple está ligado ante todo al trabajo respecto del Otro que cada pulsión produce en el cuerpo, y al lugar que allí desempeña el objeto: "Ninguna pulsión es simplemente la vuelta de otra, son disimétricas [...] lo esencial es aquí la función de un suplemento, de algo que en el nivel del Otro interroga lo que falta al Otro como tal y lo remedia (et qui y pare)" (Lacan, [196869], 2006: 255).

\section{LA ONTOLOGÍA KLEINIANA DE LA CARNE Y LA ANULACIÓN DEL FANTASMA EN LA REVERSIBILIDAD}

El modelo que Merleau-Ponty toma en cuenta para su lectura de la ontología de la carne que reivindica en Melanie Klein pareciera ser el de la reversibilidad consumada, lo que dificulta la posibilidad de pensar los estatutos psíquicos de la pulsión y el fantasma a partir de la alteridad que el objeto inscribe en el cuerpo. Para que el objeto malo interno permita precisar la atopía del fantasma es necesario un primer movimiento que expulse al objeto pulsional de la economía narcisista (No se encuentra esa expulsión en Klein, donde el ego es concebido en consonancia con la construcción de un mundo interno de objeto malos y buenos ([1952], 1997: 58).

La carencia en Klein de una teoría consistente del narcisismo9 se traduce en un modo empírico de situar psíquicamente a la fantasía como una positividad que opera como réplica de la realidad externa. De allí que el modelo kleiniano se base en la introyección de una positividad, como lo recuerda Beretta (2017: 55), donde lo introyectado (de la misma forma que aquello que se transfiere o proyecta) pareciera ser siempre algo concreto y positivo: el seno bueno o el seno malo, la madre o el padre en tanto personas. 
El modo en que es leída la interacción con el mundo es una suerte de extensión deducida del acople reversible entre el cuerpo y el mundo: "Hay pues una interacción constante entre el mundo del objeto interno que refleja de una manera fantástica las impresiones obtenidas del afuera, y el mundo externo, que está decisivamente influenciado por proyección" (Klein, [1952], 1997: 59). La dilucidación de la problemática desde una perspectiva de orden estructural, a partir de la consideración de los efectos de la inserción del objeto en un orden heterogéneo al narcisismo, permite por el contrario dotar al fantasma de una negatividad que limita esa reversibilidad absoluta a la que aspira el "poder global y universal de incorporación” (Merleau-Ponty, citado en Baas, 2011: 114).

La postulación por Merleau-Ponty de una ontología de la carne apoyada en los desarrollos kleinianos, más que permitirnos rescatar el límite a la reversibilidad que decanta de su propia distinción entre carne del cuerpo y carne del mundo, lleva más bien a disolver la primera en la segun$\mathrm{da}$ (lo que trae el consiguiente e indeseable efecto de borrar la operación específica de la negatividad en el cuerpo, cuestión clave para despejar cómo se constituye el cuerpo pulsional en psicoanálisis). Esto impacta, a su vez, en la concepción psíquica del fantasma, el que parece, al recoger reversiblemente lo que incorpora del exterior, quedar degradado a una íntima interioridad del psiquismo (como si en este movimiento se perdiera la negatividad que fundamenta al fantasma, y que permite pensar lo propio de su lugar en el entrecruce entre lo pulsional y lo narcisista).

La incorporación kleiniana puede situarse como una extensión interna, espe- cie de réplica que el psiquismo permite de la realidad del objeto externo. El cuerpo que decanta de estos abordajes es un cuerpo acoplado reversiblemente al mundo a partir de las operatorias simétricas de la proyección y la introyección. Al plantear a la reversibilidad de la pulsión a partir de una adherencia en que se borrarían los límites entre lo interior y lo exterior (2011: 116), Baas no hace otra cosa que anular la trascendencia del objeto, colocando a la problemática del cuerpo pulsional y fantasmático (abordada desde Lacan) en serie con la ontología de la carne kleiniana.

Tal vez la fascinación de Merleau-Ponty por las teorizaciones kleinianas pudo haberse debido al hecho de que nos exponen magistralmente un cuerpo que no parece estar separado del mundo por un corte, que es una de las tesis que el fenomenólogo defiende con el término indivisión en "El ojo y el espíritu" y en Lo visible y lo invisible. En la búsqueda de pensar el trabajo de la negatividad en el cuerpo en relación con la operatoria de un corte, hemos intentando mostrar el suplemento que puede extraerse de su texto, más allá de lo que aparentemente transmiten lo explícito de sus formulaciones.

\section{CONCLUSIONES}

La distinción entre la carne del cuerpo y la carne del mundo permite situar al fracaso en la reversibilidad como lo decisivo para vislumbrar el trabajo de la negatividad en el cuerpo, cuestión fundamental para circunscribir desde el psicoanálisis, en interlocución con los abordajes merleau-pontyanos, lo específico del cuerpo pulsional en su anclaje fantasmático. 
En el ámbito de lo que Merleau-Ponty interroga como narcisismo de la visión (que, como vimos, no se superpone con lo que nombramos en psicoanálisis con este mismo término) pudimos interrogar las particularidades de un cuerpo que manifiesta, en su no coincidencia consigo mismo, una alteración de esa mismidad que se construye en el registro psicoanalítico del imaginario especular. La indivisión entre actividad y pasividad, que caracteriza a la carne del mundo, colapsa en el plano del cuerpo propio, mostrando la no reversibilidad del sí mismo.

Lo que allí se inscribe como defecto puede ser problematizado en relación con el estatuto que posee para el cuerpo el objeto caduco de la pulsión parcial. A la reciprocidad sin resto entre el vidente y su imagen en el plano de la constitución del yo opusimos, pues, la otra dimensión de lo corporal, en exceso y en defecto respecto de la especularidad narcisista. Ella nos permitió precisar el estatuto del cuerpo pulsional en el eje fantasmático, más allá del límite real del autoerotismo y de las derivas sublimatorias de lo corporal.

A partir del recorrido efectuado, tomamos además en consideración la réplica de su ontología de la carne que Merleau-Ponty encuentra en el modelo kleiniano de las fantasías. En contraposición a esta versión de la carne, insistimos en la importancia de rescatar, para vislumbrar la raíz pulsional del cuerpo en su anclaje fantasmático, el fracaso de la reversibilidad que se evidencia con las consideraciones sobre la carne del cuerpo.

\section{NOTAS AMPLIATORIAS}

1. La espacialidad topológica que Merleau-Ponty promueve nos permite delinear algunos aspectos cruciales del cuerpo pulsional, aún cuando existan, como lo sostiene Lacan, ciertos puntos fundamentales del campo analítico irreductibles a su sistema. De los cuales el más notorio remite a la imposibilidad de pensar la constitución del objeto fetiche, como lo apunta en su artículo homenaje al filósofo publicado en Les Temps Modernes (1961). Este límite responde a su confinamiento en un más acá de algunos de los horizontes problemáticos abiertos por la noción de fantasma en psicoanálisis: "Si el significante del ser sexuado puede ser así desconocido en el fenómeno, es por su posición doblemente oculta (celée) en el fantasma, o sea por no indicarse sino allí donde no actúa o por no actuar más que por su falta" (Lacan, 1961: 250). Lo planteado posteriormente en La lógica del fantasma, aún luego de la fervorosa acogida de la obra póstuma del fenomenólogo en el seminario 11, viene a ratificar en parte esa idea. Allí Lacan insiste en los límites de Merleau-Ponty para situar lo fundamental de la mirada, y enuncia que el abordaje fenomenológico que efectúa en su última obra no termina por resolver "la relación de visión" (Lacan, 1966-67: 520).

2. "El espacio euclidiano es el modelo del ser perspectivo, es un espacio sin trascendencia, positivo, red de rectas, paralelas entre sí o perpendiculares, según las tres dimensiones, que alcanza todos los emplazamientos posibles". El espacio topológico es aquel "sobre el cual el pensamiento regresivo choca sin poder deducirlo directa o indirectamente [...] es un residuo perpetuo - No se encuentra solamente al nivel del mundo físico, sino que [...] funda, en fin, el principio salvaje del logos [...] el que interviene a todos los niveles para superar los problemas 
de la ontología clásica" (Merleau-Ponty, 1964: 260).

3. En este punto es preciso aclarar, ante el recurso a la banda de Moebius al que a veces se apela para pensar una mancomunidad entre los espacios topológicos de Merleau-Ponty y Lacan, que dicha banda expone una figura de la continuidad, que podríamos asimilar a una reversibilidad merleau-pontyana sin tope, continuidad en la que faltaría aún el corte producido por la sustracción del objeto.

4. A este aplastamiento lo encontramos por ejemplo en la búsqueda que Guillermo Maci emprende para articular lo parcial con lo narcisista. A este respecto, enuncia confusamente: “[...] A través de la problemática del objeto parcial lo que se advierte es que el objeto es una relación narcisista” (1987: 115).

5. Cf. "El cogito y la conciencia: lo escópico y las particularidades del mundo onírico" (Carignano, 2017).

6. Freud lo indica al decir que la etapa narcisista es relevada por la del vínculo con el objeto (Freud, [1915], 1986: 131).

7. “[...] En su versión 'activa', [la pulsión] es movimiento hacia un objeto, mientras que en su versión 'pasiva' es medio para mantener la proximidad narcisista, es una formación narcisista” (Assoun, 2001: 45).

8. Creemos que la expresión de Lacan que enuncia que "el sujeto se hace objeto amable" ([1964a], 1973: 298), y con la que pretende dar cuenta de ese destino imaginario de la transferencia que supone una degradación del Otro a un plano de espejismo narcisista, puede tener algunos inconvenientes. No hemos por ello de darle el mismo valor y alcance al hacerse en cuestión: hacerse objeto amable en este sentido transferencial del amor supone un corrimiento, un trabajo en un plano muy diferente al de la dimensión pulsional-fantasmática del hacerse.
9. "No hay en Melanie Klein ni teoría de lo imaginario ni teoría del ego" afirmaba de modo contundente Lacan en el seminario Los escritos técnicos de Freud. ([1953-54], 1975: 97). La inconsistencia que Klein apunta cuando polemiza con Freud en sus visiones respecto del narcisismo y el autoerotismo, parece estar dada por el modo de leer esas diferenciaciones, deducidas de la polarización que establece entre autoerotismo-narcisismo y relación de objeto. Cf. Klein, M. ([1952], 1997)

\section{REFERENCIAS BIBLIOGRÁFICAS}

Assoun: P-L. (2001). Le regard et la voix: leçons de psychanalyse. Paris, Anthropos.

BAAs, B. (2011). "La elaboración fenomenológica del objeto $a$ ”, en Lutereau, L. y Kripper, A (comps.), Arqueología de la mirada: Merleau-Ponty y el psicoanálisis. Buenos Aires, Letra Viva. 93123.

Beretta, A. (2017). "La voz de una carne desconocida y secreta (La voz y el instante de la angustia)", en Psicoanálisis en la Universidad, 1. Rosario, Facultad de Psicología, UNR. 53-70.

Carignano, B. (2017). "El cogito y la conciencia: lo escópico y las particularidades del mundo onírico", en Psicoanálisis en la Universidad, 1. Rosario, Facultad de Psicología, UNR. 71-90.

Duportail, G.-F. (2011). "El quiasma de una amistad: Lacan y Merleau-Ponty", en Lutereau, L. y Kripper, A (Eds.), Arqueología de la mirada: Mer- 
leau-Ponty y el psicoanálisis. Buenos Aires, Letra Viva. 71-92.

Freud, S. [1915] (1986). "Pulsiones y destinos de pulsión”, Obras completas, Buenos Aires, Amorrortu Editores, Volumen XIV, 105-134.

KLEIN, M. ([1952], 1997). "On the origins of transference", en Envy and Gratitude, and Other Works. London, Vintage.

LACAN, J. [1953-54] (1975). Les écrits techniques de Freud. Paris, Seuil.

(1958-59). Le désir et son interprétation. URL staferla.free.fr/S6/ S6.htm

(1961) "Maurice Merleau-Ponty", en Les temps modernes, 184-185, 243-254.

[1962-63] (2004). L'angoisse. Paris, Seuil.

[1964a] (1973). Les quatre concepts fondamentaux de la psychanalyse. Paris, Seuil Points.

(1964b) Fondements. URL staferla.free.fr/S11/S11.htm [1964c] (1987). Los cuatro conceptos fundamentales del psicoanálisis. Buenos Aires, Paidós.

(1966-67) La logique du fantasme. URL http://staferla.free.fr/S14/ S14.htm

[1968-69] (2006). D’un Autre à l'autre. Paris, Seuil.

MAcI, G. (1987). El objeto y el Otro. Rosario, Fundación Ross.

Merleau-Ponty, M. (1961). "L'oeil et l'esprit", en Les temps modernes, 184185, 193-227.

(1964). Le visible et l'invisible. Paris, Gallimard.
RESEÑA CURRICULAR DEL AUTOR

Bruno Carignano es psicoanalísta, Doctorando en Cotutela por convenio entre la Université Paris Diderot -Comue Sorbonne Paris Cité, Centre de recherches psychanalyse, médecine et société (CRPMS)- y la Universidad Nacional de Rosario, Facultad de Psicología, Secretaría de Estudios de Posgrados, Doctorado en Psicología. Jefe de Trabajos Prácticos concursado de Psicoanálisis II, Facultad de Psicología, UNR. Profesor Titular concursado en UCES, en las cátedras Psicoanálisis Freud II, Psicoanálisis Escuela Francesa, Taller de Trabajo Integrador Final y Psicopatología II. 